\title{
Clinical Effect of Single-Site Laparoscopy in the Treatment of Meckel's Diverticulum in Children
}

\author{
Chen Jiarong, Huang Jinging, Shi Qunfeng, Zeng Danping \\ Department of Pediatrics, Guangxi Maternal and Child Health Hospital, Nanning, P. R. China
}

Email address:

68577150@qq.com (Chen Jiarong)

\section{To cite this article:}

Chen Jiarong, Huang Jingjing, Shi Qunfeng, Zeng Danping. Clinical Effect of Single-Site Laparoscopy in the Treatment of Meckel's Diverticulum in Children. Journal of Surgery. Vol. 8, No. 1, 2020, pp. 34-37. doi: 10.11648/j.js.20200801.17

Received: February 16, 2020; Accepted: February 24, 2020; Published: March 2, 2020

\begin{abstract}
Purpose: To observe the clinical effect of laparoscopic surgery on Meckel's diverticulum in children with (MD). Method: The clinical data of 20 children were analyzed retrospectively. All children were treated with laparoscopic surgery at single part of the umbilical cord, and the operation, clinical effects and postoperative complications were observed. Result: All the 20 children completed the operation successfully, and the operation time was $50.5 \% .2 \pm 5.4$ ) there were no complications such as abdominal pain, abdominal distension, vomiting, bleeding, intestinal adhesion obstruction, intestinal stricture and intestinal anastomotic fistula during and after min,. During the follow-up of $1 \sim 26$ months, all the children ate and defecated normally and there was no recurrence. Conclusion: Umbilical laparoscopy is effective in the treatment of pediatric MD with less trauma, simple operation, short operation time and quick recovery after operation. Background Meckel's diverticulum is the most common congenital developmental malformation of the gastrointestinal tract, which is a mesenteric malformation caused by embryonic yolk tube degeneration. The incidence in the population is $2.0 \%$, most patients have no clinical symptoms, occasionally found in autopsy, laparotomy, clinical diagnosis is more difficult. The clinical manifestations of its complications are easy to be confused with other diseases such as intussusception, colonic polyps, necrotizing enteritis and acute appendicitis perforation. Date sources Based on the progress in the treatment of Meckel's diverticulum in children at home and abroad, the clinical data of 20 children with Meckel's diverticulum treated in our hospital were analyzed retrospectively to observe the clinical effect of laparoscopy-assisted operation in the treatment of Meckel's diverticulum in children. Results All the 20 children completed the operation successfully, and the operation time was 50.5\%.2 \pm 5.4 ) there were no complications such as abdominal pain, abdominal distension, vomiting, bleeding, intestinal adhesion obstruction, intestinal stricture and intestinal anastomotic fistula during and after min,. During the follow-up of $1 \sim 26$ months, all the children ate and defecated normally and there was no recurrence. Conclusions Surgical resection is the first choice for symptomatic Meckel diverticulum in children, and laparoscopic surgery is the first choice. The operative method of laparoscopic treatment of Meckel diverticulum in children with single part of umbilical cord has the advantages of less trauma, simple operation, short operation time and quick recovery after operation, and the operative effect is good.
\end{abstract}

Keywords: Laparoscopy, Meckel's Diverticulum, Children, Single-part

\section{Introduction}

Meckel's diverticulum, MD, also known as distal ileal diverticulum, is a congenital intestinal malformation caused by residual yolk tube in early embryo, with an incidence of $1-2 \%$, which is $2-4$ times higher in males than in females [1-2]. The common complications of MD are intestinal obstruction, rectal bleeding, diverticulitis, peptic ulcer perforation and so on [3]. Most of the children with MD do not have any symptoms. $8 \% \sim 22 \%$ of the children can be found because of their complications or other intestinal operations. Although B-ultrasound, CT and technetium-99m radionuclide examination have greatly improved the positive rate of MD, the preoperative diagnosis of MD is still challenging. Transumbilical laparoscopic exploration can directly observe the condition of children with MD, and it can be treated by operation at the same time of diagnosis, which has certain advantages. In this study, we retrospectively analyzed the clinical data of 20 children with MD treated by single-site laparoscopic surgery in our department, in order to provide 
reference for the diagnosis and treatment of children with MD. The report is as follows.

\section{Method}

\subsection{Clinical Data}

The clinical data of 20 children with MD treated in our department from December 2015 to November 2019 were analyzed retrospectively, including 14 boys and 6 girls, aged from 37 days to 14 years and 2 months, with an average of $1.0 \pm 0.0$. 10 cases had recurrent bloody stool, 7 cases had recurrent abdominal pain without fever, 2 cases had abdominal pain with fever, 1 case had abdominal pain with intestinal obstruction, 12 cases were positive by radionuclide scan before operation, and 20 cases were diagnosed by intraoperative exploration. Diagnostic criteria [4]: the intestinal mucosa-like structure protruding from the intestinal cavity could be seen on the contralateral margin of the mesentery at the end of the ileum. Exclusion criteria: intestinal repetitive malformation (intestinal mucosa-like structure with independent intestinal cavity in the ipsilateral margin of mesentery or intestinal cavity).

\subsection{Operation Method}

All the children underwent laparoscopic exploration of the umbilical cord and indwelled gastric tube before operation. During the operation, all the children were given general anesthesia by endotracheal intubation and supine position. Take $5 \mathrm{~mm}$ incision on the right side of umbilical chakra, insert pneumoperitoneum needle, establish carbon dioxide pneumoperitoneum (pressure is maintained at $8 \sim 10 \mathrm{mmHg}$ ), insert $5 \mathrm{~mm}$ trocar, and insert $5 \mathrm{~mm}$ laparoscope through Trocar. Then cut $5 \mathrm{~mm}$ on the left side of the umbilical wheel, opened the umbilical wheel fascia, directly inserted the $5 \mathrm{~mm}$ non-invasive grip under laparoscopy (see figure 1), explored the celiac intestinal canal, and sequentially explored the small intestinal canal to the end of the ileum. It can be seen that the intestinal mucous membrane-like structure with protruding intestinal cavity on the contralateral edge of the mesentery is $\mathrm{MD}$; fixed with MD clamp. Remove the pneumoperitoneum and the Trocar, on the right side of the umbilical wheel to connect the incision on both sides of the umbilical wheel to form a semicircular incision on the upper edge of the umbilical cord (if the intestinal edema and adhesion is serious, the incision can be appropriately extended to the left and right sides), and the MD of the clamp is raised out of the abdominal cavity through the incision on the superior edge of the umbilical cord (see figure 2). Then the intestine at $2.5 \mathrm{~cm}$ away from MD and MD was resected and sent for pathological examination, and the end-to-end anastomosis of mucous layer was performed with $5 / 0$ or $4 / 0$ absorbable line, and the seromuscular layer was strengthened and sutured. The intestinal inflation test showed that the intestinal tube was unobstructed without fistula and stricture, and the diameter of the anastomosis could pass through the adult index fingertip. Suture closed the mesenteric defect (see figure 3), and the intestinal canal was returned to the abdominal cavity in turn. The peritoneum and superficial fascia were closed with 4 / 0 absorbable thread, and the incision at the upper edge of the umbilical cord was sutured with 5 / 0 absorbable thread (see figure 4). Fasting, gastrointestinal decompression and antibiotics to prevent infection for 5 days after operation, liquid diet was given on the 5th day after operation, and returned to normal diet on the 7 th day after operation.

\subsection{Observation Index}

The time of operation, the occurrence of intraoperative and postoperative complications (including bleeding, intestinal injury, abdominal pain, abdominal distension, vomiting, intestinal adhesion obstruction, intestinal stricture and intestinal anastomotic fistula, etc.) were observed, and the postoperative outpatient follow-up was performed to understand the defecation, eating, growth and development and recurrence of the children. All the 20 children completed the operation successfully, and the operation time was $50.5 \%$. $2 \pm 5$. 4) min. Postoperative pathological examination showed that abnormal gastric mucosa-like structure was found in 9 cases of MD, pancreatic mucosa-like structure in 1 case of MD and ileal mucosa-like structure in the other 10 cases of MD. MD was diagnosed pathologically. There was no massive hemorrhage and intestinal accessory injury during the operation, no symptoms such as abdominal pain, abdominal distension, vomiting and bleeding, and no obvious complications such as intestinal adhesion obstruction, intestinal stricture and intestinal anastomotic fistula. During the follow-up of $1 \sim 35$ months, all the children had normal eating and defecation, normal weight gain, no recurrence of abdominal pain and bloody stool, and no recurrence of MD.

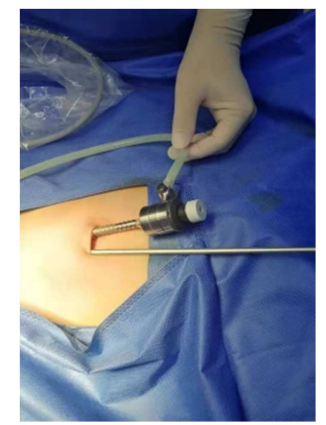

Figure 1. Laparoscopic incision on both sides of umbilical chakra.

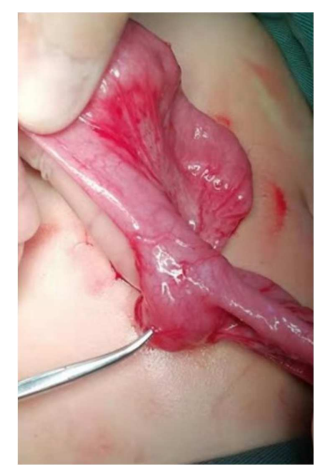

Figure 2. Pull MD out of abdominal cavity. 


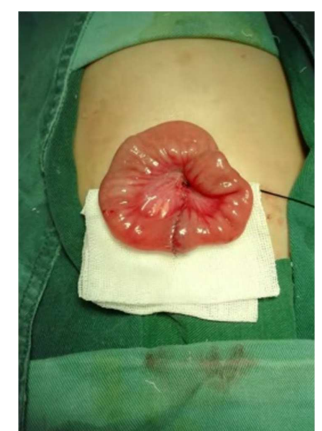

Figure 3. MD was excised and intestinal anastomosis was performed.

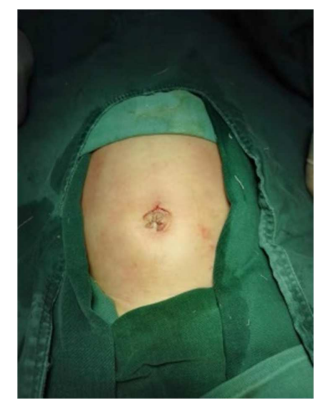

Figure 4. Umbilical incision after suture.

\section{Result}

All the 20 children completed the operation successfully,

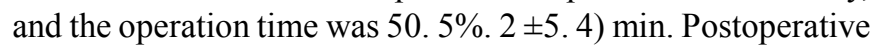
pathological examination showed that gastric mucosal-like structure was found in 8 cases of resected MD, pancreatic mucosal-like structure in 1 case of resected MD, ileal mucosal-like structure in other cases of MD. MD was diagnosed pathologically. There was no massive hemorrhage and intestinal accessory injury during the operation, no symptoms such as abdominal pain, abdominal distension, vomiting and bleeding, and no complications such as intestinal adhesion obstruction, intestinal stricture and intestinal anastomotic fistula. During the follow-up of $1 \sim 28$ months, all the children had normal eating and defecation, normal weight gain, no recurrence of abdominal pain and bloody stool, and no recurrence of MD.

\section{Discussion}

MD is caused by incomplete degeneration of yolk tube and unclosed intestinal end. It is common in infants, and there are no specific signs in abdomen. Gastrointestinal radiography may not show positive findings [4]. The clinical manifestations of MD complications are easy to be confused with other diseases such as intussusception, colonic polyps, necrotizing enteritis and acute appendicitis perforation, so it is easy to be misdiagnosed and missed. Abdominal pain and bloody stool are the main symptoms of MD. Complications occur in every age group, from infants to the elderly, but they mainly affect children. A comprehensive analysis of MD, Żyluk Andrzej [20] shows that most surgeons decide to remove $\mathrm{MD}$, during surgery, even if it is asymptomatic, to prevent possible further complications. Radionuclide scanning is an important method for the diagnosis of MD, which can greatly improve the preoperative diagnosis rate of MD with bleeding as the main clinical manifestation [5-7]. However, radionuclide scan has a certain false positive rate, including intestinal obstructive disease, intussusception and inflammatory diseases [8-10], and the false negative diagnosis of MD without bleeding is higher [11]. With the improvement of B-ultrasound technology in recent years, the diagnosis rate of B-ultrasound has also been greatly improved. For cases with hemorrhage and diverticulitis, the coincidence rate of diagnosis can be as high as $88.1 \%$. However, the final clinical diagnosis still needs surgical exploration [12].

Surgical resection is the first choice for symptomatic MD in children, and laparoscopic surgery is the first choice [21]. The traditional exploratory laparotomy has a certain degree of blindness, and the surgical injury is large, the recovery is slow, and the parents should not accept it. Laparoscopic exploration has been paid more and more attention because of its advantages such as simple operation, small incision, quick recovery and less postoperative complications. At present, there are many methods for laparoscopic treatment of MD, including basal ligation [13], wedge resection [14], partial intestinal resection and end-to-end anastomosis, intestinal stapler resection [15], ultrasonic knife resection [16-17] and so on. It is generally believed that wedge resection of diverticulum and transverse suture of intestine are the first choice for the treatment of Meckel's diverticulum [18]. However, the key to the operation lies in the complete removal of ectopic mucosa. Some scholars divide MD into simple type and complex type [16], simple type MD has the same structure as surrounding intestinal tissue, no inflammation, can be directly wedge-shaped resection; complex type MD basal tissue edema, stiffness, scar, or diverticulum gangrene, or MD, located in the ileal mesenteric region need partial intestinal resection and anastomosis, and need to be quickly frozen pathological analysis during the operation to determine the appropriate degree of resection. However, each method has its own advantages and disadvantages: (1) Basal ligation is only suitable for MD, with narrow diameter of basal intestinal canal, but there is a risk of recurrence and intestinal fistula caused by residual ectopic mucosa, (2) wedge resection is simple, short operation time, good intestinal blood circulation, not easy to have intestinal fistula, but there may also be residual ectopic mucosa. (3) Intraabdominal intestinal resection and anastomosis, ultrasonic knife resection and anastomosis and intestinal stapler resection may cause abdominal contamination, and laparoscopy takes a long time and requires skilled operation skills. (4) Extraabdominal diverticulectomy is put forward through the extended incision of the navel after the diverticulum is found under laparoscope, and resection and anastomosis is performed outside the abdominal cavity, which reduces the pollution of the abdominal cavity, reduces the cost of hospitalization, and the diverticulum is resected completely. Therefore, at present, the resection of extraabdominal diverticulum is widely used in clinic. (5) the stapler is expensive and not suitable for younger children [19]. 
(6) it takes a long time to wait for the results of frozen section during operation, which increases the risk of anesthesia. In this study, single-site laparoscopic surgery was used to treat MD. After the diagnosis of MD was confirmed by laparoscopy, MD was raised outside the abdominal cavity for intestinal resection and intestinal anastomosis without contaminating the abdominal cavity. There was no anastomotic leakage and intestinal adhesion obstruction on the 5th day after operation. During the postoperative follow-up, the eating and defecation of all the children were normal, and there was no recurrence of MD.

\section{Acknowledgements}

To sum up, Surgical resection is the first choice for symptomatic Meckel diverticulum in children, and laparoscopic surgery is the first choice. Single-site laparoscopic surgery for the treatment of pediatric MD, has the advantages of less trauma, simple operation, short operation time and quick recovery after operation, and the effect is good, which is worth popularizing. Although there are various methods of laparoscopic treatment of Meckel's diverticulum in children, as long as the doctor is skilled and follows the medical principle, it is a good means of treatment.

\section{References}

[1] Mackey WC, Dineen P. A fifty year experience with Meckel's diverticulum [J]. Surg Gynecol Obstet, 1983, 156 (1): 56-64.

[2] St-Vil D, Brandt ML, Panic S, et al. Meckel's diverticulum in children: a 20-year review [J]. J Pediatr Surg, 1991, 26 (11): 1289-1292.

[3] Bubnjar J. Meckel's diverticulitis in the early postoperative course after appendectomy [J]. Acta Med Croatica, 2013, 67 (1): 65-68.

[4] She Yaxiong. Pediatric surgery [M]. Beijing: people's Health Publishing House, 1995: 136-137.

[5] Blevrakis E, Partalis N, Seremeti C, et al. Meckel's diverticulum in paediatric practice on Crete (Greece): a 10-year review [J]. Afr J Paediatr Surg, 2012, 8 (3): 279- 282.

[6] Sinha CK, Pallewatte A, Easty M, et al. Meckel's scan in children: a review of 183 cases referred to two paediatric surgery specialist centres over 18 years [J]. Pediatr Surg Int, 2013, 29 (5): 511- 517.

[7] Menezes M, Tareen F, Saeed A, et al. Symptomatic Meckel's diverticulum in children: a 16-year review [J]. Pediatr Surg Int, 2008, 24 (5): 575-577.
[8] Rho JH, Kim JS, Kim SY, et al. Clinical Features of Symptomatic Meckel's Diverticulum in Children: Comparison of Scintigraphic and Non-scintigraphic Diagnosis [J]. Pediatr Gastroenterol Hepatol Nutr, 2013, 16 (1): 41-48.

[9] Pampal A, Aksakal ED. Littre hernia in childhood: a case report with a brief review of the literature [J]. Afr J Paediatr Surg, 2011, 8 (2): 221-224.

[10] Rangarajan M, Palanivelu C, Senthilkumar R, et al. Laparoscopic surgery for perforation of Meckel's diverticulum [J]. Singapore Med J, 2007, 48 (4): e102-e105.

[11] Sagar J, Kumar V, Shah DK. Meckel's diverticulum: a systematic review [J]. J R Soc Med, 2006, 99 (10): 501-505.

[12] Fu Xiuting, Wu Mingjun, Liu Ziwei, Liu Chang, Tian Ye. Value of Ultrosound in Diagnosis of Complications of Meckel's Diverticulum in Children [J]. Heilongiiang Medicine, 2019 (11): 1385.

[13] Xu Wei, Deng Qingqiang, et al. Laparoscopic resection of Meckel's diverticulum in children with intraoperative frozen base ligation: a report of 43 cases. Jiangxi Medicine, 2016, 51 (12): 1306-1308.

[14] Chan KW, Lee KH, Mou JW, et al. Laparoscopic management of complicated Meckel's diverticulum in children: a 10-year review [J]. Surg Endosc, 2008, 22 (6): 1 509-1 512.

[15] Palanivelu C, Rangarajan M, Senthilkumar R, et al. Laparoscopic management of symptomatic Meckel's diverticula: a simple tangential stapler excision [J]. JSLS, 2008, 12 (1): 66-70.

[16] Duan X, Ye G, Bian H, et al. Laparoscopic vs. laparoscopically assisted management of Meckel's diverticulum in children [J]. Int J Clin Exp Med, 2015, 8 (1): 94-100.

[17] Yang Jun, Guo Qin, Duan Xufei, et al. Total laparoscopic resection of Meckel's diverticulum. Operation [J]. Chinese Journal of General surgery, 2012 Magazine 27 (9): 733-735.

[18] Kong Chihuan, Ma Jidong. A review of the diagnosis and treatment of Meckel's diverticulum (report of 39 cases) [J]. Journal of Clinical Pediatric surgery, 2015, 4 (6): 444-446.

[19] Homero Rivas, Wang Yongkun, Zhu Jiangfan. Single-hole laparoscopic surgery: endoscopic hand. New advances in surgery $[\mathrm{J}]$. Chinese Journal of minimally invasive surgery, 2009, 15 (12): 1063-1065.

[20] Żyluk Andrzej, Management of incidentally discovered unaffected Meckel's diverticulum-a review.[J]. Pol Przegl Chir 2019 Aug 12; 91 (6): 41-46.

[21] Keese Daniel, Rolle Udo, Gfroerer Stefan et al. Symptomatic Meckel's Diverticulum in Pediatric Patients-Case Reports and Systematic Review of the Literature. Front Pediatr, 2019; 7. 